\title{
Optimal head rotation and puncture site for internal jugular vein cannulation after laryngeal mask airway insertion
}

\author{
Won Ho $\underline{K i m}^{1}$, MD, Mi Sook $\underline{G w a k}^{2}$, MD, PhD, Soo Joo $\underline{\mathrm{Choi}}^{2}$, MD, PhD, Sang Hoon Song${ }^{2}$, MD, Myung Hee $\underline{K i m}^{2}$, MD, PhD
}

\begin{abstract}
INTRODUCTION We studied the effect of head rotation on the relative position of the right common carotid artery (CCA) and the right internal jugular vein (IJV) in patients with laryngeal mask airway (LMA) insertion to evaluate the accuracy of anatomical landmarks for right IJV cannulation.

METHODS We simulated needle insertion to the right IJV on sonograms via the central landmark approach and an approach using the external jugular vein, in patients with LMA insertion $(n=50)$ or endotracheal intubation (E-tube, $n=50$ ). Overlap index and successful simulation rates were measured according to the different degrees of head rotation. RESULTS The overlap index between the right CCA and the right IJV increased with greater degrees of head rotation. It was significantly greater in the LMA insertion group than in the E-tube group in the following head rotation positions: neutral, $15^{\circ}$ and $45^{\circ}$. The success rate of the simulation was lower in the LMA insertion group than in the E-tube group. In the LMA insertion group, the success rate of the simulation was highest (62\%) with the central landmark approach and in the $15^{\circ}$ head rotation position.

CONCLUSION In the LMA insertion group, the overlap index increased incrementally with greater head rotation degrees (from neutral to $45^{\circ}$ ). The central landmark approach and $15^{\circ}$ head rotation position appear to be the optimal puncture site and degree of head rotation for right IJV cannulation in patients with LMA insertion.
\end{abstract}

Keywords: central venous cannulation, jugular veins, laryngeal mask airway, ultrasonography

\section{INTRODUCTION}

Recently, the laryngeal mask airway (LMA) is frequently used for patients under general anaesthesia. ${ }^{(1-7)}$ Occasionally, a clinical situation that requires internal jugular vein (IJV) cannulation after LMA insertion is encountered, especially for difficult airways in patients undergoing major surgery. ${ }^{(8,9)}$ However, the anatomic relationship of the IJV and the common carotid artery (CCA) has been reported to change significantly in patients after LMA insertion. ${ }^{(10)}$ The degree of overlap between the right IJV and the right CCA was found to be greater after LMA insertion. ${ }^{(10)}$ In our experience, there are many cases of complete overlap between the right IJV and the right CCA after LMA insertion. This means that there is a higher risk of puncturing the CCA and a lower rate of successful IJV cannulation, when IJV cannulation is performed after LMA insertion. Lieberman et al reported that an increase in head rotation was associated with a higher probability of CCA contact; ${ }^{\text {;11 }}$ we postulated that the effect of head rotation would be similar in patients with LMA insertion. If the degree of overlap between the right IJV and the right CCA varies according to the degree of head rotation after LMA insertion, the degree of head rotation with the least amount of overlap can be determined and the risk of CCA puncture can therefore be reduced.

The central landmark approach, which is commonly used for IJV cannulation, was shown to have a low success rate in patients with LMA insertion. ${ }^{(12)}$ Takeyama et al recommended the use of a lower puncture point - near the area where the clavicular head of the sternocleidomastoid muscle (SCM) attaches to the clavicle; the CCA was not observed to be in the vicinity of the IJV when the patient was in that position even after LMA insertion. ${ }^{(10)}$ Although the authors performed test punctures at the supraclavicular lower puncture point in 20 patients without any complications, Kim et $\mathrm{al}^{(13)}$ opined that the supraclavicular puncture could still be associated with pneumothorax, hydrothorax or cardiac tamponade. Takeyama et al evaluated the puncture point using only a $30^{\circ}$ head rotation, ${ }^{(10)}$ without evaluating the effect of different degrees of head rotation. Another commonly used landmark approach for IJV cannulation is the skin point $1.5 \mathrm{~cm}$ or $2.5 \mathrm{~cm}$ medial to the external jugular vein (EJV). ${ }^{(14)}$ The use of this landmark has not been evaluated in patients with LMA insertion.

In the present study, we compared the degrees of overlap between the right IJV and the right CCA as well as the success rates of simulated right IJV cannulation between patients with LMA insertion and patients with endotracheal intubation (E-tube) using ultrasonography. We aimed to find: (a) the optimal degree of head rotation for right IJV cannulation; and (b) a suitable skin landmark for right IJV cannulation after LMA insertion. To achieve these goals, we compared the success rates of simulated right IJV cannulation at different degrees of head rotation and used different skin landmarks for right IJV cannulation.

\section{METHODS}

This study was registered at www.clinicaltrials.gov (registration no. NCT01407302) and approved by the Institutional Review Board of Samsung Medical Centre, Korea (2011-04-024-001). Written informed consent was obtained from all patients included in this study.

${ }^{1}$ Department of Anesthesiology and Pain Medicine, Samsung Changwon Hospital, Sungkyunkwan University School of Medicine, Changwon, ${ }^{2}$ Department of Anesthesiology and Pain Medicine, Samsung Medical Center, Sungkyunkwan University School of Medicine, Seoul, Republic of Korea

Correspondence: Dr Mi Sook Gwak, Professor, Department of Anesthesiology and Pain Medicine, Samsung Medical Center, Sungkyunkwan University School of Medicine, 50 Irwon-Dong, Gangnam-Gu, Seoul 135-710, Republic of Korea. misookgwak@gmail.com 
A total of 100 patients undergoing any type of surgery under general anaesthesia with LMA insertion $(n=50)$ or E-tube ( $n=50$ ) were enrolled in this prospective observational study. Patients with any known abnormal neck anatomy, previous surgery or trauma involving the neck, prior cannulation of neck vessels, predicted or confirmed difficult airway, or any dental problems were excluded from the study. The H-Universal Stand, with a 6-MHz transducer and a two-dimensional image display (Sonosite, Bothell, WA, USA), was used for ultrasonography.

Anaesthesia was induced using $5 \mathrm{mg} / \mathrm{kg}$ sodium pentothal and $0.8 \mathrm{mg} / \mathrm{kg}$ rocuronium. In the LMA insertion group, the LMA Classic $^{\text {TM }} 5.0$ or 4.0 (Laryngeal Mask Company Ltd, Westmeath, Ireland) was used, while in the E-tube group, an 8.0- or 7.0-mm endotracheal tube was used $(8.0 \mathrm{~mm}$ for men and $7.0 \mathrm{~mm}$ for women). The LMA cuff volume was adjusted to achieve a cuff pressure between $50 \mathrm{mmHg}$ and $55 \mathrm{mmHg}$ (measured using a cuff pressure gauge). The tidal volume was adjusted to $8 \mathrm{~mL} / \mathrm{kg}$ and the respiratory rate was adjusted to maintain a partial pressure of carbon dioxide between $35 \mathrm{mmHg}$ and $45 \mathrm{mmHg}$.

The primary outcome variable was the overlap index of the right IJV and the right CCA, ${ }^{(15)}$ which was measured using sonograms and according to the degree of head rotation from the midline $\left(0^{\circ}, 15^{\circ}, 30^{\circ}\right.$ and $\left.45^{\circ}\right)$. The overlap index was derived from the ratio of the overlap length of the right IJV to the horizontal diameter of the right CCA. The calculation formula is as follows: overlap index $=($ right IJV overlap length $[\mathrm{mm}] /$ right CCA horizontal diameter $[\mathrm{mm}]) \times 100 \%$ (Fig. 1). ${ }^{(15)}$ All other measurements taken are shown in Fig. 1.

To determine a suitable skin landmark for right IJV cannulation in patients with LMA insertion, we evaluated the central ${ }^{(16,17)}$ and right EJV landmarks. ${ }^{(14)}$ Needle insertion was simulated using the ultrasonography length measurement tool to indicate the simulated needle pathway in the sonogram (Fig. 2). To simulate the central landmark approach, ${ }^{(16,17)}$ we placed a surgical pen mark at the apex of the triangle formed by the two heads (sternal and clavicular) of the SCM on the skin of the right neck (Fig. 2). The right EJV was located at the level of the cricoid cartilage in the sonogram. ${ }^{(14)}$ With ultrasonography guidance, the simulation line was drawn vertically from the skin mark in the central landmark approach, and $1.5 \mathrm{~cm}$ and $2.5 \mathrm{~cm}$ medial to the medial border of the right EJV in the EJV approach; the line was then evaluated for right IJV and right CCA puncture (Fig. 3). The simulation line was drawn to extend from the tip of the ultrasound transducer and to pass through the neck. CCA puncture was defined as the intersection of any part of the simulation line with any part of the inner lumen of the CCA. IJV puncture was defined as the intersection of the simulation line with the central $80 \%$ of the inner lumen of the IJV, as lateral-sided puncture of the IJV often results in the failure of central line catheter insertion. Successful IJV cannulation was defined as the intersection of the simulation line with the middle $80 \%$ of the IJV and no intersection with the CCA.

We did not perform any intervention to increase the area of the right IJV (e.g. Valsalva manoeuvre or Trendelenburg position). The investigator held the ultrasound transducer in a manner similar to that of holding a syringe for needle insertion. The transducer was

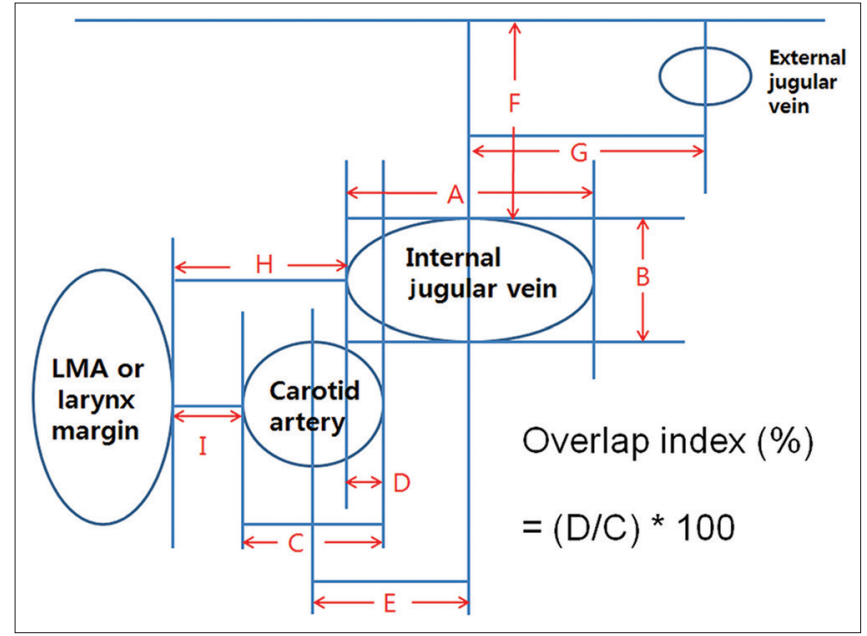

Fig. 1 Diagram shows the measurements made on the sonograms. A: horizontal diameter of the internal jugular vein (IJV); B: vertical diameter of the right IJV; C: diameter of the right common carotid artery (CCA); $\mathrm{D}$ : overlap distance between the right CCA and the right IJV; E: distance between the centre of the right CCA and centre of the right IJV; F: depth of the right IJV from the skin; G: distance between the centre of the right IJV and centre of the external jugular vein; $\mathrm{H}$ : distance from lateral margin of the laryngeal mask airway (LMA) or larynx to the right IJV; I: distance from the lateral margin of the LMA or larynx to the right CCA.

directed $30^{\circ}$ caudad within the parasagittal plane (Fig. 2). Minimal pressure was used to hold the transducer so as not to compress the right IJV. ${ }^{(18)}$ An experienced investigator drew the skin markings and positioned the ultrasound transducer, while another investigator performed the ultrasonographic measurements. The investigator holding the transducer was not aware of the findings. To reduce any bias related to data collection, all measurements were taken twice and the average data was used in the analysis.

The measurements were taken with the patient's head rotated through four positions: $0^{\circ}$ (head midline), and $15^{\circ}, 30^{\circ}$ and $45^{\circ}$ to the left of the midline (Fig. 2). The skin landmarks were evaluated according to the different head positions. When the patient's sagittal plane was perpendicular to the operation table, the degree of head rotation was set at $0^{\circ}$. A protractor with a guidance bar was used to align the sagittal plane to the desired head rotation degree (Fig. 2). The patient's head was rotated to adjust the nasal midline to the guidance bar of the protractor. For each approach (i.e. central landmark and right EJV landmark) of IJV cannulation, the incidence of successful simulation was measured for each degree of head rotation.

This study was powered to detect a minimum difference of $20 \%$ in the overlap index between the LMA insertion group and the E-tube group, with an alpha error of $5 \%$ and a beta error of $80 \%$. Based on a pilot study we conducted, a standard deviation of $34 \%$ in the overlap index of patients with LMA insertion was used in the calculations. To detect a $20 \%$ difference in the overlap index between the groups, 45 patients were needed in each group. Assuming a dropout rate of $10 \%$, 50 patients were needed in each group.

Kolmogorov-Smirnov tests with Lilliefors correction were used to assess the normality of the data distribution. Continuous variables were compared between the two groups using either unpaired $t$-test or the Mann-Whitney $U$ test, depending on the 


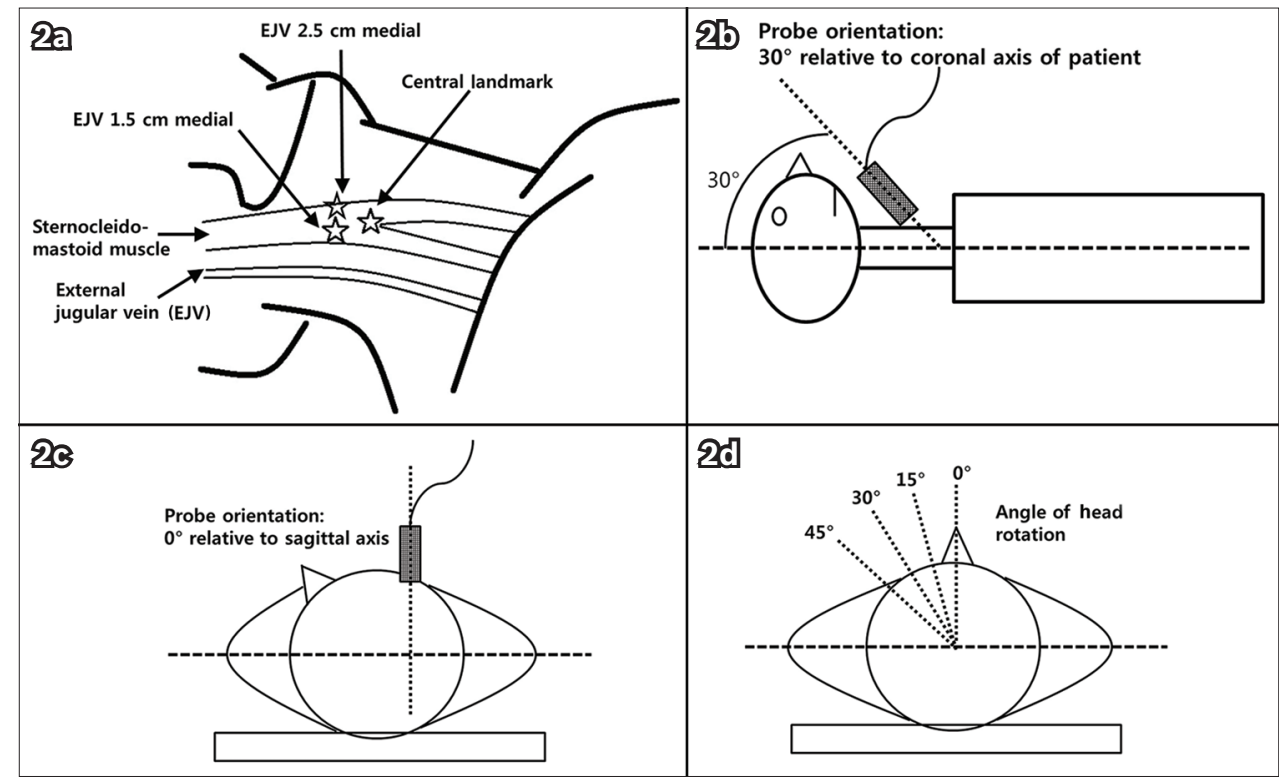

Fig. 2 Illustrations show how measurements were made using an ultrasound transducer. (a) Location of the skin mark (is) for central landmark approach relative to the sternocleidomastoid muscle (SCM) was drawn at the apex of the triangle that is formed by the two heads (sternal and clavicular) of the SCM. (b) The ultrasound transducer was directed $30^{\circ}$ caudad. (c) The transducer was positioned parallel to the sagittal plane of the patient. (d) The patient's head was rotated left from the midline position $\left(0^{\circ}\right)$ to $45^{\circ}$, in $15^{\circ}$ increments.

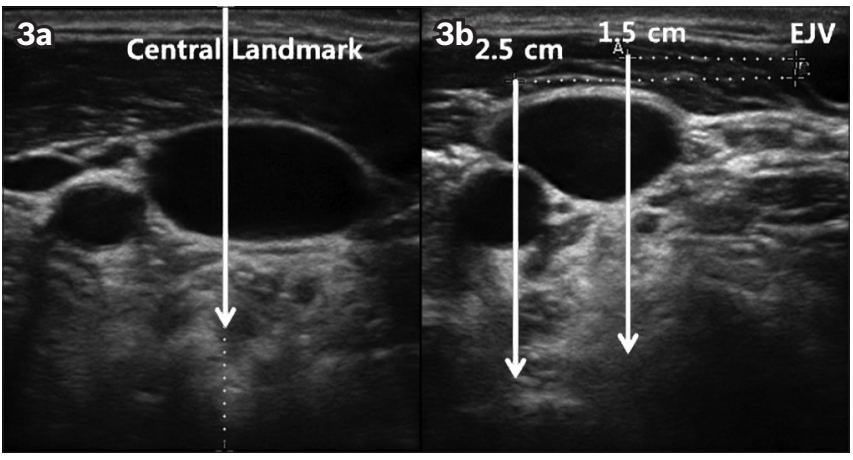

Fig. 3 Representative sonograms show the simulation line (for simulation of right IJV cannulation) drawn (a) vertically from the skin mark made for the central landmark approach; and (b) $1.5 \mathrm{~cm}$ and $2.5 \mathrm{~cm}$ medial to the medial border of the right external jugular vein.

normality of the variables. Chi-square test or Fisher's exact test was used to compare the success rates of IJV cannulation between the two groups, according to their expected counts. Comparisons of variables among the same patients were done using repeated measures analysis of variance (ANOVA). Comparisons of variables taken at different time points for the same patients were done using paired $t$-test or the Wilcoxon signed-rank test, depending on the normality of the variables. $p<0.05$ was considered statistically significant. All data analyses were performed using the IBM SPSS Statistics version 20.0 (IBM Corp, Armonk, NY, USA).

\section{RESULTS}

No data was missing for the 100 patients enrolled in this study. No adverse events or complications occurred in any of the patients. The demographic data of the patients is shown in Table I. There was no significant difference between the demographic characteristics of patients in the LMA insertion group and those in the E-tube group.
Table I. Demographic data of the patients $(n=100)$.

\begin{tabular}{|c|c|c|c|}
\hline \multirow[t]{2}{*}{ Variable } & \multicolumn{2}{|c|}{ Mean \pm standard deviation } & \multirow[t]{2}{*}{ p-value } \\
\hline & $\begin{array}{l}\text { E-tube group } \\
\quad(n=50)\end{array}$ & $\begin{array}{l}\text { LMA insertion group } \\
\qquad(\mathrm{n}=50)\end{array}$ & \\
\hline Age (yr) & $43 \pm 10$ & $40 \pm 14$ & 0.236 \\
\hline Gender ${ }^{+}$ & & & 0.260 \\
\hline Male & $39(78.0)$ & $34(68.0)$ & \\
\hline Female & $11(22.0)$ & $16(32.0)$ & \\
\hline Weight (kg) & $68 \pm 12$ & $73 \pm 16$ & 0.085 \\
\hline Height $(\mathrm{cm})$ & $168 \pm 6$ & $169 \pm 11$ & 0.721 \\
\hline $\begin{array}{l}\text { Body mass } \\
\text { index }\left(\mathrm{kg} / \mathrm{m}^{2}\right)\end{array}$ & $23.8 \pm 3.6$ & $25.2 \pm 4.0$ & 0.059 \\
\hline $\begin{array}{l}\text { Body surface } \\
\text { area }\left(\mathrm{m}^{2}\right)\end{array}$ & $1.77 \pm 0.18$ & $1.84 \pm 0.25$ & 0.136 \\
\hline
\end{tabular}

Both the right IJV and the right CCA were present and patent on ultrasonography in all patients and for all head positions. Table II shows the ultrasonographic measurements for both groups. There were significant increases from baseline in the overlap distance for the $15^{\circ}, 30^{\circ}$ and $45^{\circ}$ head rotation positions in the LMA insertion group, and for $30^{\circ}$ and $45^{\circ}$ head rotation positions in the E-tube group. In both groups, the overlap index increased significantly from baseline in the $15^{\circ}, 30^{\circ}$ and $45^{\circ}$ head rotation positions (LMA insertion group - baseline: $25 \% \pm 24 \%$ vs. neutral: $41 \% \pm 31 \%, 15^{\circ}$ head rotation: $62 \% \pm 37 \%, 30^{\circ}$ head rotation: $71 \% \pm 34 \%, 45^{\circ}$ head rotation: $82 \% \pm 31 \%$; $\mathrm{p}=0.093, \mathrm{p}<0.001, \mathrm{p}<0.001, \mathrm{p}<0.001$, respectively). There were significant differences between the groups in the overlap distance, and the distance between the centre of the right CCA and the centre of the right IJV, in the neutral and the $15^{\circ}$ head rotation positions. There were also significant differences between the groups in the distance between the centre of the 
Table II. Ultrasonographic measurements for the endotrachea intubation (E-tube) group and the laryngeal mask airway (LMA) insertion group.

\begin{tabular}{|c|c|c|c|}
\hline \multirow[t]{2}{*}{ Measurement } & \multicolumn{2}{|c|}{ Mean \pm standard deviation } & \multirow[t]{2}{*}{ p-value* } \\
\hline & $\begin{array}{l}\text { E-tube group } \\
\quad(n=50)\end{array}$ & $\begin{array}{l}\text { LMA insertion } \\
\text { group }(n=50)\end{array}$ & \\
\hline \multicolumn{4}{|l|}{ Baseline } \\
\hline $\mathrm{A}(\mathrm{cm})$ & $1.62 \pm 0.59$ & $1.57 \pm 0.51$ & 0.659 \\
\hline $\mathrm{B}(\mathrm{cm})$ & $0.98 \pm 0.36$ & $0.94 \pm 0.34$ & 0.655 \\
\hline $\mathrm{C}(\mathrm{cm})$ & $0.69 \pm 0.12$ & $0.67 \pm 0.09$ & 0.208 \\
\hline $\mathrm{D}(\mathrm{cm})$ & $0.18 \pm 0.16$ & $0.17 \pm 0.16$ & 0.597 \\
\hline $\mathrm{E}(\mathrm{cm})$ & $1.05 \pm 0.19$ & $1.00 \pm 0.20$ & 0.220 \\
\hline $\mathrm{F}(\mathrm{cm})$ & $1.04 \pm 0.14$ & $1.04 \pm 0.15$ & 0.834 \\
\hline $\mathrm{G}(\mathrm{cm})$ & $1.51 \pm 0.94$ & $1.61 \pm 0.95$ & 0.599 \\
\hline Overlap index (\%) & $23 \pm 18$ & $25 \pm 24$ & 0.650 \\
\hline \multicolumn{4}{|l|}{ Neutral position } \\
\hline $\mathrm{A}(\mathrm{cm})$ & $1.64 \pm 0.47$ & $1.62 \pm 0.37$ & 0.755 \\
\hline $\mathrm{B}(\mathrm{cm})$ & $1.00 \pm 0.44$ & $0.98 \pm 0.23$ & 0.844 \\
\hline $\mathrm{C}(\mathrm{cm})$ & $0.72 \pm 0.16$ & $0.70 \pm 0.22$ & 0.487 \\
\hline $\mathrm{D}(\mathrm{cm})$ & $0.19 \pm 0.15$ & $0.28 \pm 0.22$ & $0.019^{+}$ \\
\hline$E(\mathrm{~cm})$ & $1.03 \pm 0.18$ & $0.92 \pm 0.29$ & $0.017^{\dagger}$ \\
\hline $\mathrm{F}(\mathrm{cm})$ & $1.05 \pm 0.17$ & $1.03 \pm 0.17$ & 0.462 \\
\hline $\mathrm{G}(\mathrm{cm})$ & $1.58 \pm 0.85$ & $1.77 \pm 0.69$ & 0.205 \\
\hline Overlap index (\%) & $24 \pm 15$ & $41 \pm 31$ & $0.001^{+}$ \\
\hline \multicolumn{4}{|l|}{$15^{\circ}$ head rotation } \\
\hline$A(\mathrm{~cm})$ & $1.61 \pm 0.43$ & $1.59 \pm 0.37$ & 0.806 \\
\hline $\mathrm{B}(\mathrm{cm})$ & $0.95 \pm 0.37$ & $0.97 \pm 0.26$ & 0.744 \\
\hline $\mathrm{C}(\mathrm{cm})$ & $0.69 \pm 0.13$ & $0.66 \pm 0.10$ & 0.233 \\
\hline $\mathrm{D}(\mathrm{cm})$ & $0.30 \pm 0.24$ & $0.40 \pm 0.23$ & $0.046^{+}$ \\
\hline$E(\mathrm{~cm})$ & $0.92 \pm 0.32$ & $0.71 \pm 0.31$ & $0.001^{+}$ \\
\hline $\mathrm{F}(\mathrm{cm})$ & $1.01 \pm 0.20$ & $0.98 \pm 0.20$ & 0.535 \\
\hline $\mathrm{G}(\mathrm{cm})$ & $1.61 \pm 0.85$ & $1.72 \pm 0.69$ & 0.454 \\
\hline Overlap index (\%) & $39 \pm 28$ & $62 \pm 37$ & $0.001^{+}$ \\
\hline \multicolumn{4}{|l|}{$30^{\circ}$ head rotation } \\
\hline$A(\mathrm{~cm})$ & $1.53 \pm 0.45$ & $1.55 \pm 0.39$ & 0.800 \\
\hline $\mathrm{B}(\mathrm{cm})$ & $0.92 \pm 0.35$ & $0.93 \pm 0.26$ & 0.866 \\
\hline$C(\mathrm{~cm})$ & $0.66 \pm 0.13$ & $0.64 \pm 0.09$ & 0.268 \\
\hline $\mathrm{D}(\mathrm{cm})$ & $0.43 \pm 0.25$ & $0.44 \pm 0.21$ & 0.875 \\
\hline $\mathrm{E}(\mathrm{cm})$ & $0.66 \pm 0.13$ & $0.60 \pm 0.33$ & $0.038^{+}$ \\
\hline $\mathrm{F}(\mathrm{cm})$ & $0.99 \pm 0.25$ & $0.96 \pm 0.20$ & 0.523 \\
\hline $\mathrm{G}(\mathrm{cm})$ & $1.64 \pm 0.86$ & $1.63 \pm 0.67$ & 0.931 \\
\hline Overlap index (\%) & $60 \pm 26$ & $71 \pm 34$ & 0.079 \\
\hline \multicolumn{4}{|l|}{$45^{\circ}$ head rotation } \\
\hline $\mathrm{A}(\mathrm{cm})$ & $1.46 \pm 0.41$ & $1.45 \pm 0.36$ & 0.965 \\
\hline$B(\mathrm{~cm})$ & $0.90 \pm 0.29$ & $0.91 \pm 0.23$ & 0.912 \\
\hline$C(\mathrm{~cm})$ & $0.61 \pm 0.35$ & $0.63 \pm 0.09$ & 0.407 \\
\hline $\mathrm{D}(\mathrm{cm})$ & $0.47 \pm 0.26$ & $0.51 \pm 0.20$ & 0.387 \\
\hline$E(\mathrm{~cm})$ & $0.62 \pm 0.35$ & $0.46 \pm 0.30$ & $0.016^{+}$ \\
\hline $\mathrm{F}(\mathrm{cm})$ & $0.94 \pm 0.24$ & $0.98 \pm 0.21$ & 0.448 \\
\hline $\mathrm{G}(\mathrm{cm})$ & $1.56 \pm 0.82$ & $1.52 \pm 0.63$ & 0.835 \\
\hline Overlap index (\%) & $69 \pm 31$ & $82 \pm 31$ & $0.048^{+}$ \\
\hline
\end{tabular}

* Result of unpaired $t$-test or the Mann-Whitney $U$ test. + Statistically significant difference. A: horizontal diameter of the right internal jugular vein (IJV); B: vertical diameter of the right IJV; C: diameter of the right common carotid artery (CCA); $\mathrm{D}$ : overlap distance between the right CCA and the right IJV; E: distance between the centre of the right CCA and centre of the right IJV; F: depth of the right IJV from the skin; G: distance between the centre of the right IJV and centre of the external jugular vein (Fig. 1)

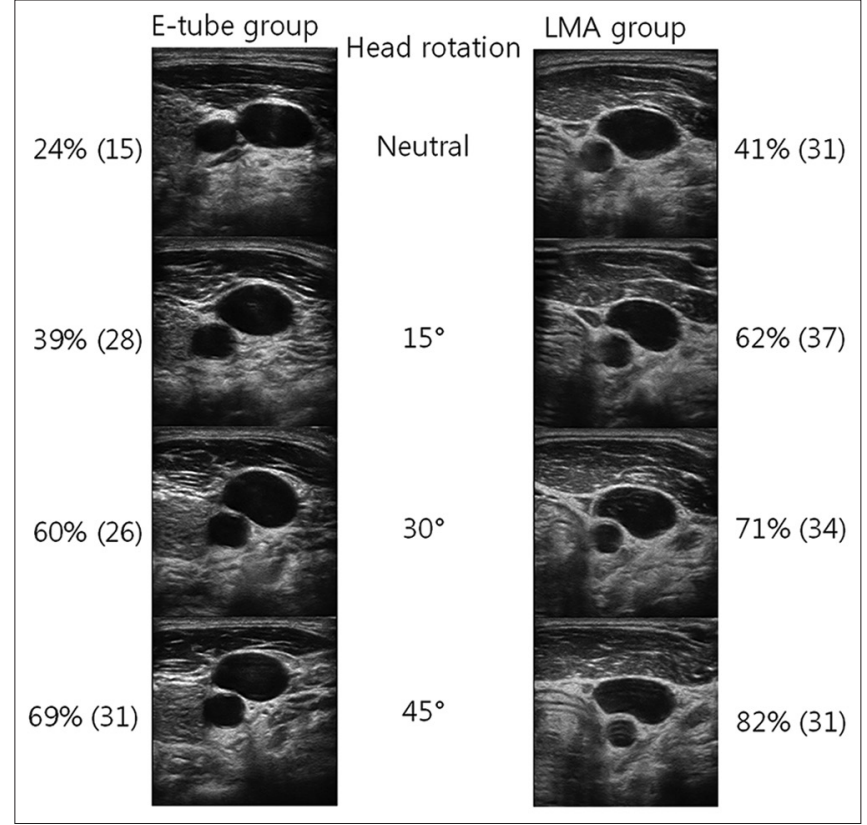

Fig. 4 Sonograms show overlap between the right internal jugular vein and the right common carotid artery among patients with laryngeal mask airway (LMA) insertion and endotracheal intubation (E-tube) at different degrees of head rotation. The values indicate overlap indices (mean [standard deviation]).

CCA and the centre of the IJV in the $30^{\circ}$ and $45^{\circ}$ head rotation positions. There were significant differences between the groups in overlap indices in the neutral position, and the $15^{\circ}$ and $45^{\circ}$ head rotation positions $\left(15^{\circ}\right.$ head rotation: $39 \pm 28 \%$ in the E-tube group vs. $62 \pm 37 \%$ in the LMA insertion group, $p=0.001)$. The mean values of the overlap indices, as well as the representative sonograms, for both groups according to the degree of head rotation are shown in Fig. 4.

Table III and Fig. 5 show the incidences of simulated right CCA puncture and right IJV puncture, and the success rates of simulated right IJV cannulation in both groups. The values were tabulated according to the different landmarks for right IJV cannulation. The rate of right CCA puncture increased and the success rate of simulated right IJV cannulation decreased with a greater degree of head rotation. There were significant differences in the success rates of simulated right IJV cannulation between the two groups in the neutral position, and in the $30^{\circ}$ and $45^{\circ}$ head rotation positions, when the central landmark approach was used. When the skin landmark $1.5 \mathrm{~cm}$ medial to the right EJV was used, there were also significant differences in the success rates of simulated right IJV cannulation between the two groups in the neutral position, and in the $15^{\circ}, 30^{\circ}$ and $45^{\circ}$ head rotation positions. Significant differences in the success rates of simulated right IJV cannulation between the two groups were observed in the neutral position, and in the $30^{\circ}$ and $45^{\circ}$ head rotation positions, when the skin landmark $2.5 \mathrm{~cm}$ medial to the right EJV was used. In the LMA insertion group, the success rate of simulated IJV cannulation was highest (62.0\%) using the central landmark approach in the $15^{\circ}$ head rotation position. In the E-tube group, the success rate was highest (80.0\%) using the central landmark approach in the neutral position or $15^{\circ}$ head rotation position. 
Table III. Simulated common carotid artery (CCA) and internal jugular vein (IJV) puncture rates and successful IJV cannulation rate according to the different degrees of head rotation in the endotracheal intubation (E-tube) group and the laryngeal mask airway (LMA) insertion group.

\begin{tabular}{|c|c|c|c|c|c|c|c|}
\hline \multirow[t]{2}{*}{ Position } & \multicolumn{3}{|c|}{ E-tube group $(n=50)$} & \multicolumn{3}{|c|}{ LMA insertion group $(n=50)$} & \multirow[t]{2}{*}{ p-value* } \\
\hline & $\begin{array}{c}\text { CCA } \\
\text { puncture }\end{array}$ & $\begin{array}{c}\text { IJV } \\
\text { puncture }\end{array}$ & $\begin{array}{l}\text { Success } \\
\text { rate }\end{array}$ & $\begin{array}{c}\text { CCA } \\
\text { puncture }\end{array}$ & $\begin{array}{c}\text { IJV } \\
\text { puncture }\end{array}$ & $\begin{array}{l}\text { Success } \\
\text { rate }\end{array}$ & \\
\hline Neutral & $6(12.0)$ & $43(86.0)$ & $40(80.0)$ & $8(16.0)$ & $35(70.0)$ & $30(60.0)$ & 0.049 \\
\hline $15^{\circ}$ & $7(14.0)$ & $42(84.0)$ & $40(80.0)$ & $7(14.0)$ & $32(64.0)$ & $31(62.0)$ & 0.077 \\
\hline $45^{\circ}$ & $21(42.0)$ & $32(64.0)$ & $25(50.0)$ & $26(52.0)$ & $20(40.0)$ & $4(8.0)$ & $<0.001$ \\
\hline \multicolumn{8}{|l|}{ EJV 1.5} \\
\hline Neutral & $2(4.0)$ & $38(76.0)$ & $36(72.0)$ & $2(4.0)$ & $25(50.0)$ & $24(48.0)$ & 0.024 \\
\hline $15^{\circ}$ & $7(14.0)$ & $35(70.0)$ & $29(58.0)$ & $9(18.0)$ & $21(42.0)$ & $16(32.0)$ & 0.015 \\
\hline $30^{\circ}$ & $11(22.0)$ & $34(68.0)$ & $24(48.0)$ & $13(26.0)$ & $21(42.0)$ & $11(22.0)$ & 0.011 \\
\hline $45^{\circ}$ & $10(20.0)$ & $30(60.0)$ & $20(40.0)$ & $12(24.0)$ & $18(36.0)$ & $9(18.0)$ & 0.027 \\
\hline Neutral & $10(20.0)$ & $32(64.0)$ & $27(54.0)$ & $14(28.0)$ & $25(50.0)$ & $13(26.0)$ & 0.008 \\
\hline $15^{\circ}$ & $11(22.0)$ & $28(56.0)$ & $21(42.0)$ & $11(22.0)$ & $20(40.0)$ & $13(26.0)$ & 0.139 \\
\hline $30^{\circ}$ & $14(28.0)$ & $24(48.0)$ & $16(32.0)$ & $16(32.0)$ & $15(30.0)$ & $6(12.0)$ & 0.028 \\
\hline $45^{\circ}$ & $18(36.0)$ & $18(36.0)$ & $13(26.0)$ & $13(26.0)$ & $11(22.0)$ & $2(4.0)$ & 0.004 \\
\hline
\end{tabular}

Data presented as no. (\%). * Result of chi-square test or Fisher's exact test comparing success rates between groups. EJV 1.5: skin landmark approach that is $1.5 \mathrm{~cm}$ medial to the medial border of the right external jugular vein (EJV); EJV 2.5: skin landmark approach that is $2.5 \mathrm{~cm}$ medial to the medial border of the right EJV

\section{DISCUSSION}

In the present study, we compared the overlap index between the right CCA and the right IJV among patients with LMA insertion or E-tube, according to different degrees of head rotation. To the best of our knowledge, this is the first study to compare patients with LMA insertion to those with E-tube. We found that the overlap index was greater in the LMA insertion group than in the E-tube group for all degrees of head rotation evaluated in this study. The landmarks for right IJV cannulation in patients with LMA insertion were also evaluated; the success rates of right IJV cannulation using these landmarks were compared between patient with LMA insertion and those with E-tube. The success rates of right IJV cannulation were significantly lower in the LMA insertion group than in the E-tube group for both the central landmark and EJV-guided approaches. The success rates were higher when the central landmark approach was used and highest when it was used in a $15^{\circ}$ head rotation position.

After LMA insertion, cannulation of the right IJV may be more difficult (it frequently results in right CCA puncture) because of the overlap between the IJV and CCA, and the displacement of the SCM (which makes the central landmark less valuable for IJV puncture and palpating the CCA more difficult). ${ }^{(19)}$ Sulek et al ${ }^{(15)}$ studied the effect of head rotation on the overlap index between the carotid artery and the IJV in 12 volunteers without E-tube. The authors found that the mean overlap of CCA with IJV was $45 \%$ in an $80^{\circ}$ head rotation position. ${ }^{(15)}$ Lieberman et $\mathrm{al}^{(11)}$ reported that an increased head rotation to the left of the midline was associated with a higher probability of a simulated needle contacting the IJV or CCA; they also concluded that the head should be rotated no more than $30^{\circ}$ in patients with high body mass indices (BMIs) or high body surface areas (BSAs) when attempting IJV cannulation. Interestingly, in Lieberman et al's study, increased BSAs and BMIs were associated with a higher probability of CCA contact in $45^{\circ}$ and $60^{\circ}$ head rotation positions. ${ }^{(11)}$ However, the present study's data showed no significant correlation between the success rate of IJV cannulation and BSAs or BMIs using any landmark or in any degree of head rotation.

In the present study, the overlap index between the right IJV and right CCA increased with greater degrees of head rotation. This was observed for both the LMA insertion group and the E-tube group. The extent of overlap was greater in the LMA insertion group than in the E-tube group for any degree of head rotation. The risk of CCA puncture was also found to be somewhat greater in the LMA insertion group than the in E-tube group; this risk tends to increase with greater degrees of head rotation.

The incidences of CCA puncture during the simulated right IJV cannulation in the present study were greater than the actual incidences previously reported $(4.2 \%-8.3 \%) .{ }^{(18,20-22)}$ This is because some of the simulated CCA punctures may not occur in real-life situations; they would occur only if a needle traversed the IJV lumen in cases where the overlap index is high. Furthermore, some needles aimed at the CCA may slide past the artery without puncturing it. It is clear, however, that the simulated CCA puncture rate is somewhat higher in the LMA insertion group than in the E-tube group.

One possible reason as to why the IJV cannulation success rate is lower, and CCA puncture rate is higher, in patients with LMA insertion than in those with E-tube is that the IJV overlaps with the CCA after LMA insertion and cuff inflation. In the present study, we found that the overlap distance increases, and the distance between the centre of the IJV and centre of the CCA decreases after LMA insertion. However, the vessel sizes of the CCA and the IJV did not differ significantly between the LMA insertion group and the E-tube group. This means that 

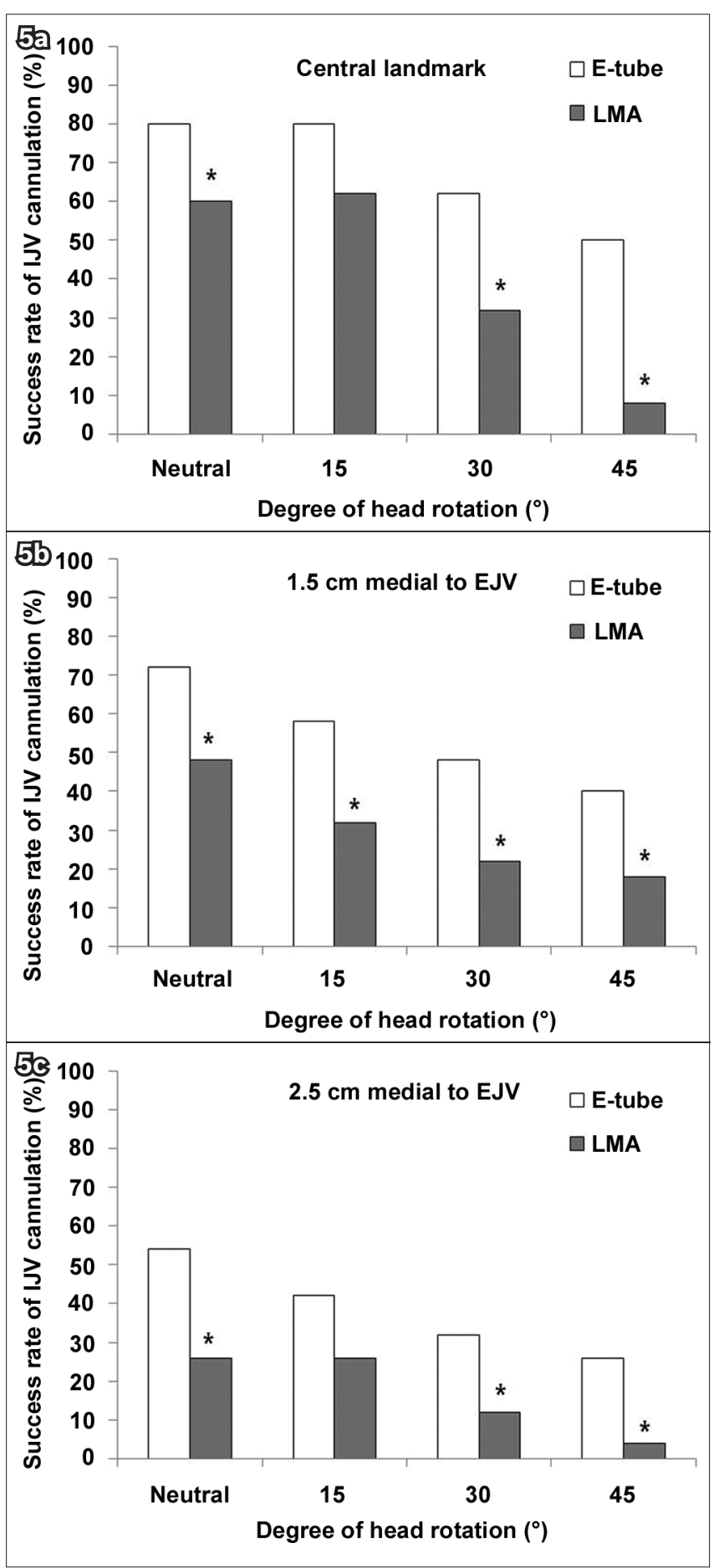

Fig. 5 Bar graphs show the effect of head rotation on the success rate of simulated right internal jugular vein (IJV) cannulation when using: (a) the central landmark; (b) the landmark $1.5 \mathrm{~cm}$ medial to the external jugular vein (EJV); and (c) the landmark $2.5 \mathrm{~cm}$ medial to the EJV. *Significant differences between groups.

the IJV and the CCA are not compressed after LMA insertion. Therefore, to increase the success rate of IJV cannulation and reduce the risk of CCA puncture, minimising the overlap index by reducing the degree of head rotation would be more effective than increasing the vessel size by preload increment or respiratory manoeuvre. Although the overlap index was smallest in the neutral head position (i.e. without rotation), the needle and catheter for IJV cannulation comes into contact with the mandible in this position, preventing the procedure.
Therefore, small degrees of head rotation should be tolerated for cannulation.

The present study has several limitations. First, as the needle passage was simulated on an ultrasonography machine monitor, the needle passage during an actual procedure could have been different. However, Bailey et al have validated this simulation method for the evaluation of IJV cannulation success rate, ${ }^{(23)}$ confirming that the simulated needle path on the sonogram using the digital cursor was identical to the path taken by an actual needle. Previous studies have also used the same simulation technique for studying IJV cannulation. ${ }^{(11,23)}$ Second, we evaluated the landmark at the cricoid cartilage or central landmark levels, and not at the high and low levels of the neck that were studied by Takeyama et al. ${ }^{(10)}$ However, we focused on examining the effect of different degrees of head rotation on the overlap between the vessels in patients with LMA insertion. Third, we measured all the outcome variables in the supine position without any intervention to increase the area of the right IJV. Previous studies reported that manoeuvres that increase preload (e.g. Trendelenburg position, Valsalva manoeuvre, liver compression and passive leg elevation) can increase the cross-sectional area of the IJV. $(24,25)$ If these manoeuvres were adopted in this study, the overlap index may have been different. Fourth, as the present study was performed in a single institution, the external validity of the study is limited. However, a direct comparison of the patients with LMA insertion and those with E-tube helps to provide an unbiased study outcome.

In conclusion, the overlap index was greater in the LMA insertion group than in the E-tube group for all degrees of head rotation evaluated in this study. In the LMA insertion group, the overlap index increased with greater degrees of head rotation (from a neutral position to a $45^{\circ}$ head rotation position). The success rate of the simulated right IJV cannulation was significantly lower in the LMA insertion group than in the E-tube group regardless of the approach used (central landmark or EJVguided). The optimal puncture site and degree of head rotation for right IJV cannulation in patients with LMA insertion appear to be the central landmark and the $15^{\circ}$ position.

\section{REFERENCES}

1. Raveendran R, Sastry SG, Wong DT. Tracheal extubation with a laryngeal mask airway and exchange catheter in a patient with a difficult airway. Can J Anaesth 2013; 60:1278-9.

2. Kammah M, Añez C. Prolonged use of the LMA Supreme ${ }^{T M}$. Can J Anaesth 2013; 60:411-2.

3. Murphy C, Wong DT. Airway management and oxygenation in obese patients. Can J Anaesth 2013; 60:929-45.

4. Ambulkar R, Tan AY, Chia NC, Low TC. Comparison between use of neuromuscular blocking agent and placebo with the intubating laryngeal mask airway. Singapore Med J 2008; 49:462-5

5. Noor Zairul M, Khairul Faizi A. Comparison of the VBM laryngeal tube and laryngeal mask airway for ventilation during manual in-line neck stabilisation. Singapore Med J 2006; 47:892-6.

6. Sharma R, Dua CK, Saxena KN. A randomised controlled study comparing the effects of laryngeal mask airway and endotracheal tube on early postoperative pulmonary functions. Singapore Med J 2011; 52:874-8.

7. Suhitharan T, Seevanayagam S, Parker FC, Teoh WH. Acute unilateral submandibular gland swelling associated with the laryngeal mask airway. Singapore Med J 2013; 54:e236-9.

8. Goldman LJ, Nodal C, Jimenez E. Successful airway control with the laryngeal mask in an infant with Beckwith-Wiedemann syndrome and hepatoblastoma 
for central line catheterization. Paediatr Anaesth 2000; 10:445-8.

9. Riley RH, Gaylard DG, Wright DA, Davies PC. The LMA and difficulty with internal jugular vein cannulation. Anaesthesia 1999; 54:1224.

10. Takeyama K, Kobayashi H, Suzuki T. Optimal puncture site of the right internal jugular vein after laryngeal mask airway placement. Anesthesiology 2005; 103:1136-41.

11. Lieberman JA, Williams KA, Rosenberg AL. Optimal head rotation for internal jugular vein cannulation when relying on external landmarks. Anesth Analg 2004; 99:982-8.

12. Tseng KY, Tsai CJ, Wu SH, et al. Accuracy of the central landmark for catheterization of the right internal jugular vein after placement of the ProSeal laryngeal mask airway. Acta Anaesthesiol Taiwan 2009; 47:118-22.

13. Kim MH, Lee DJ, Kim MC. Bilateral hydrothorax and cardiac tamponade after right subclavian vein catheterization -A case report-. Korean Anesthesiol 2010; 59 Suppl: S211-7.

14. Jin Lee J, Sook Gwak M, Yang M, Soo Kim G. A new method of internal jugular vein catheterization using the cricoid cartilage and the external jugular vein as a landmark. Am J Emerg Med 2006; 24:697-701.

15. Sulek CA, Gravenstein N, Blackshear RH, Weiss L. Head rotation during internal jugular vein cannulation and the risk of carotid artery puncture. Anesth Analg 1996; 82:125-8.

16. Daily PO, Griepp RB, Shumway NE. Percutaneous internal jugular vein cannulation. Arch Surg 1970; 101:534-6.

17. Civetta JM, Gabel JC, Gemer M. Internal-jugular-vein puncture with a margin of safety. Anesthesiology 1972; 36:622-3

18. Denys BG, Uretsky BF, Reddy PS. Ultrasound-assisted cannulation of the internal jugular vein. A prospective comparison to the external landmarkguided technique. Circulation 1993; 87:1557-62.

19. Skolnick ML. The role of sonography in the placement and management of jugular and subclavian central venous catheters. AJR Am J Roentgenol 1994; 163:291-5.

20. Troianos CA, Jobes DR, Ellison N. Ultrasound-guided cannulation of the internal jugular vein. A prospective, randomized study. Anesth Analg 1991; 72:823-6.

21. Goldfarb G, Lebrec D. Percutaneous cannulation of the internal jugular vein in patients with coagulopathies: an experience based on 1,000 attempts. Anesthesiology 1982; 56:321-3.

22. Jobes DR, Schwartz AJ, Greenhow DE, Stephenson LW, Ellison N. Safer jugular vein cannulation: recognition of arterial puncture and preferential use of the external jugular route. Anesthesiology 1983; 59:353-5.

23. Bailey PL, Whitaker EE, Palmer LS, Glance LG. The accuracy of the central landmark used for central venous catheterization of the internal jugular vein. Anesth Analg 2006; 102:1327-32.

24. Armstrong PJ, Sutherland R, Scott DH. The effect of position and different manoeuvres on internal jugular vein diameter size. Acta Anaesthesiol Scand 1994; 38:229-31.

25. Mallory DL, Shawker T, Evans RG, et al. Effects of clinical maneuvers on sonographically determined internal jugular vein size during venous cannulation. Crit Care Med 1990; 18:1269-73. 